\title{
Inveterated Facial Palsy Correction with Endoscopically Assisted Multiple Muscle Transposition and Lifts: The EMTL Procedure
}

\author{
Marco Borsetti ${ }^{1}$ Ezio Nicola Gangemi ${ }^{1,0}$ Silvia Germano ${ }^{1} \quad$ Alessandra Clemente $^{1}$ \\ Luca Devalle $^{1}$ Claudia Cerato $^{1}$ Giorgio Merlino ${ }^{1}$ \\ ${ }^{1}$ Division of Plastic Surgery, Hand Surgery and Microsurgery, \\ Surgical Department of ASL Città di Torino, Maria Vittoria Hospital, \\ Turin, Italy \\ Address for correspondence Ezio Gangemi, MD, Division of Plastic \\ Surgery, Hand Surgery and Microsurgery, Surgical Department \\ of ASL Città di Torino, Maria Vittoria Hospital, Turin, 10146, Italy \\ (e-mail: ezio.gangemi@gmail.com).
}

Indian J Plast Surg:2020;53:344-348

\begin{abstract}
Introduction The aim of the present analysis was to study the safety and efficacy associated with reanimation in facial nerve palsy by the endoscopically assisted multiple muscle transposition and lifts (EMTL).

Patients and Methods The study sample included all patients who had undergone a facial reanimation by EMTL procedure from September 2015 to May 2019. The patients were analyzed retrospectively, with more than 1 year of follow-up, and were evaluated in terms of functional-aesthetic results and postoperative complications. The outcome was evaluated with the Sunnybrook scale.

Results Fourteen patients were included in the present study. They were all inveterate palsies with minimum 4 years from the initial injury. The preoperative Sunnybrook score ranged from 0 to 5 and the postoperative ranged from 30 to 65 . Spontaneous smile achievement was obtained in 10 patients and only mild restoration in one patient. The scar and static correction were satisfactory in all patients. Eye protection

Keywords

- facial palsy

- endoscopic

- reanimation was improved in all cases with some form of active blinking in six cases.

Conclusion This study showed that facial palsy correction with EMTL procedure offers a promising alternative treatment for patients with facial palsy not suitable for microsurgical muscle transposition.
\end{abstract}

\section{Introduction}

Inveterate facial palsy can be addressed with different surgical techniques aiming at rehabilitating one or more facial units. The treatment must be individualized and cannot be easily standardized since clinical presentation and patient condition are different. There are patients that refuse microsurgical muscle transfer or patient that for comorbidities or elderly are not eligible for complex and long surgery. ${ }^{1,2}$ In such cases, procedures with limited invasiveness become a valuable tool to correct the deformity. The endoscopically assisted multiple muscle transposition and lifts (EMTL) procedure consists in a comprehensive endoscopic surgical treatment of facial palsy.

Over a 3-year period (2015-2018), 14 patients with inveterate unilateral facial paralysis had reanimation by EMTL procedure in our center.

\section{Surgical Technique}

EMTL is an association of multiple procedures in a single operation using several endoscopic portals. Three different

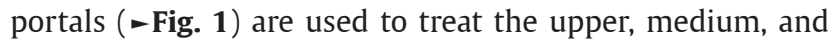
lower face plus four stab incisions. The procedure starts published online December 24, 2020
DOI https://doi.org/ 10.1055/s-0040-1721877 ISSN 0970-0358. (c) 2020. Association of Plastic Surgeons of India.

This is an open access article published by Thieme under the terms of the Creative Commons Attribution-NonDerivative-NonCommercial-License, permitting copying and reproduction so long as the original work is given appropriate credit. Contents may not be used for commercial purposes, or adapted, remixed, transformed or built upon. (https://creativecommons.org/licenses/by-nc-nd/4.0/).

Thieme Medical and Scientific Publishers Pvt. Ltd. A-12, 2nd Floor, Sector 2, Noida-201301 UP, India 


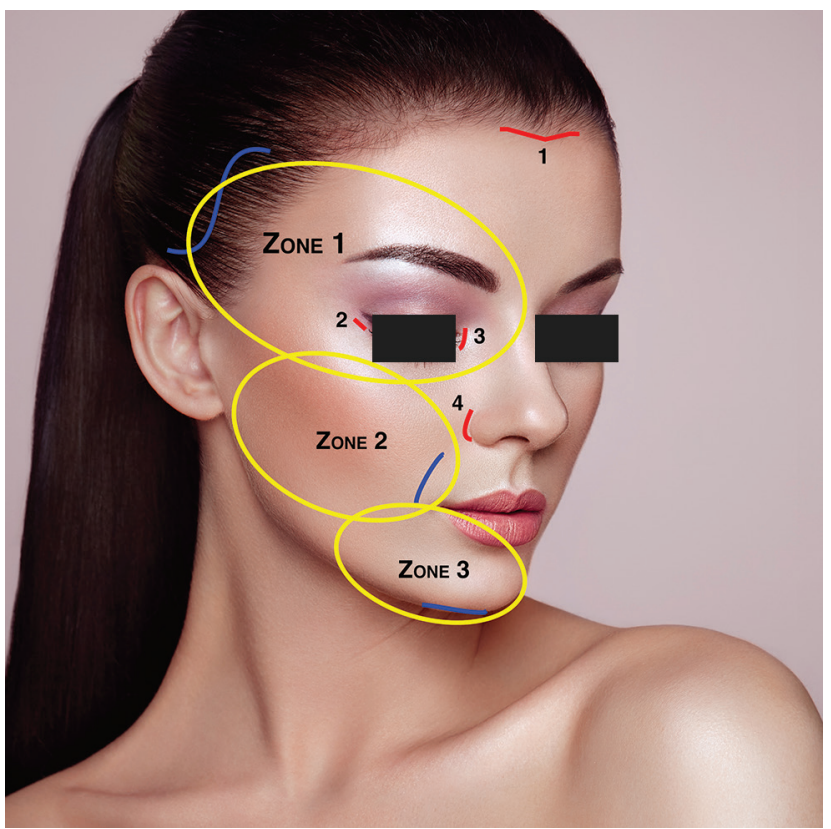

Fig. 1 Scheme of the portals and the dissection area. Portal 1: eyebrow lift, temporalis fascia graft, temporalis sling fabrication; Portal 2: modified Labbè myoplasty, ala nasi support with temporalis fascia graft; Portal 3: digastric transposition and platismoplasty. Red lines: accessory stab incision.

treating the orbital region with an endoscopic eyebrow lift and temporal sling myoplasty of the eye and goes on with portals preparation for modified Labbè myoplasty ${ }^{3,4}$ and digastric muscles transposition with platismoplasty through the same access. A strip of fascia temporalis is used to reinforce and lift the alae nasi. The temporalis muscle with its fascia is used to reanimate and to support the lower lid with a partial temporal sling.

\section{In-Depth Description of Treatment Site I: Parieto-Temporal Area}

A 3-cm parieto-temporal skin incision at the hair bearing line is used to manage a triple procedure: the eyebrow lifting, the temporalis fascia graft harvesting, and the creation of a temporalis muscle sling for the eye.

\section{Brow Lift}

Lateral temporal approach to brow lift as described by Nahai and Saltz: through the incision over the temporalis muscle the lateral orbital attachments, adhesions, and septa are released and the parieto-temporal fascia is elevated and anchored to the deep temporal fascia with lateral brown elevation. ${ }^{5}$

\section{Temporalis Fascia Graft}

A $1 \times 3 \mathrm{~cm}$ strip of deep temporal fascia is harvested and grafted to support and lift the ala nasi.

\section{Temporalis Sling (-Fig. 2)}

The temporalis muscle is harvested in its $2-\mathrm{cm}$ central portion keeping intact the cranial attachment to the parietal fascia and pedicled on the caudal area; it is then flipped
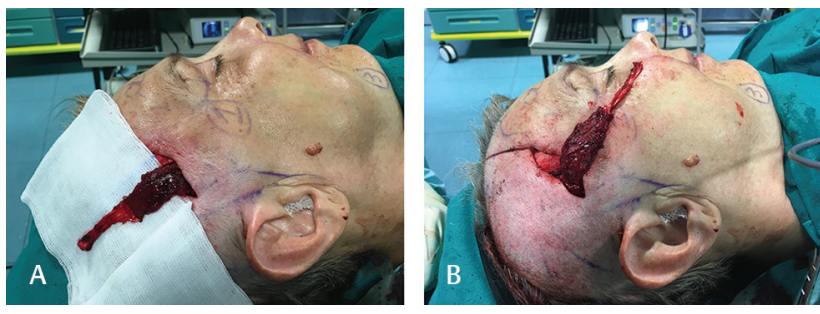

Fig. 2 Temporalis sling. (A) Harvesting. (B) Downwards rotation.
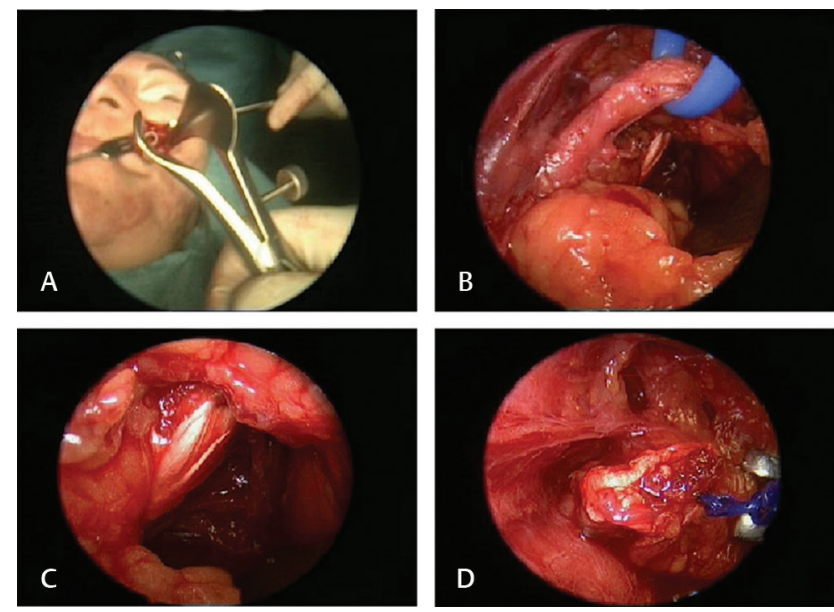

Fig. 3 Endoscopic Labbè myoplasty. (A) Preparation of the zygomatic area. (B) Isolation and preservation on Stenone duct. (C) Isolation of temporalis tendon. (D) Detachment of temporalis tendon from coronoid process.

downwards and tunneled with the fascia extension separated in two 2-mm slips which are afterwards conducted through the upper and lower lid by a needle-cannula and secured at the medial canthal region by a stab incision. ${ }^{6}$ When the temporal muscle contract it acts like a dynamic sphincter partially mimicking the blink mechanism.

\section{In-Depth Description of Treatment Site II: Nasolabial Fold}

The technique is similar to the one described by Boahene et $\mathrm{al}^{7}$ but the skin incision has been reduced through the use of endoscopy and a dedicated instrumentation.

At the nasolabial fold is created a key point portal dedicated to the modified Labbè myoplasty. A $1.5-\mathrm{cm}$ skin incision is dissected down to the zygomatic area and blunt dissection is used to expose the Bichat bubble and the parotid duct ( - Fig. 3 ). The dedicated instrumentation has been chosen to assist the dissection. It consists of a long nasal speculum, blunt scissors, 2-mm 30-degree endoscope with a soft tissue supporting valve, and a diathermy forceps ( - Fig. 4). When the safety of the duct is assured, the dissection proceeds endoscopically and under magnification all around the temporalis tendon muscle, and the coronoid process begins. The apex of coronoid process is dissected free in the subperiosteal plane keeping in continuity the temporalis tendon to the medial insertion on the mandible. A 2-mm chisel is used to perform the apex osteotomy and the tendon is detached medially 

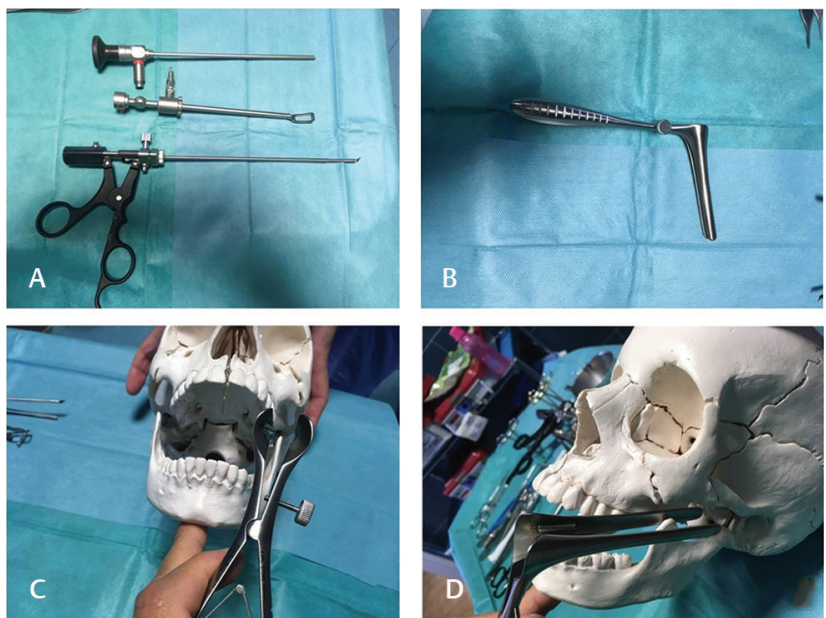

Fig. 4 Dedicated instruments for modified Labbè procedure. (A) Optical endoscopic portal, bipolar electrocautery scissors, and blunt dissector. (B) Nasal speculum. (C, D) Anterior and lateral view of the position of nasal speculum down to the zygomatic area.

and derotated to gain length. The tendon is divided and secured to the orbicularis oris and zygomaticus muscle insertion is performed in the region of the modiolus with 3-0 polydioxanone sutures. The position of the tendon insertion is determined by the preoperative smile pattern. To establish the appropriate tension on the transposed tendon, the temporalis muscle is stimulated through surface electrodes while varying traction tension on the tendon is applied.

At the end the portal is also used to support the alar base of the nose with the temporal fascia graft.

\section{In-Depth Description of Treatment Site III: Mental Area}

A 1- to 2-cm submental incision allows preparation and transfer of digastric muscle and platismoplasty. The anterior digastric muscle is isolated maintaining the innervations from the fifth cranial nerve and rotated to restore the function of the depressor labii inferioris muscle. The median tendon is driven subcutaneously to the lower lip and secured with a tiny stab incision. Conley et $\mathrm{al}^{8}$ stressed the importance of including all of the subdigastric tendon because it provides half the length of the transposed segment and permits strategic insertion into the lower lip. This was performed by splitting the end of the digastric tendon and using these two strips to suture to the orbicularis oris muscle at its middle or inferior border. Instead of submandibular approach previously described, we use a single middle submental incision and the dissection is assisted by the endoscope (-Fig. 5). At this stage if platysma contracture is present it is treated through the same portal with delivery and section of contracted fibers.

\section{Accessory Stab Incisions}

1. Incision centered in the midline in the hair bearing region: using a dedicate o-degree endoscope, the forehead is elevated in the subperiosteal plane. The supraorbital nerve and the supratrochlear nerve are identified, preserved,

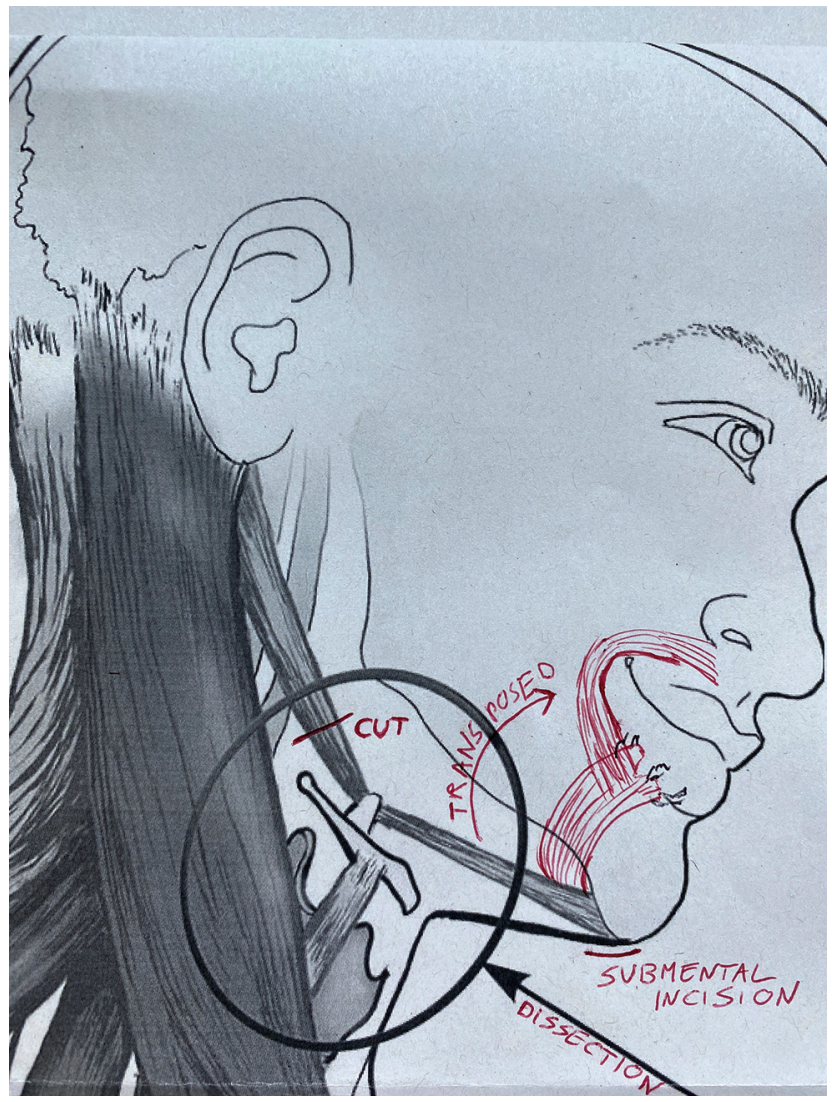

Fig. 5 Diagram of digastric transfer.

and released. The contralateral corrugators attachments are released to improve symmetry.

2. (medial) and 3. (lateral). Two periorbital incisions helping the tunneling of the temporal sling.

4. Incision located at the base of the alae nasi for temporal fascia graft positioning.

\section{Postoperative Treatments}

All patients were operated under general anesthesia and discharged the day after the procedure. Intravenous administration of antibiotics was stopped at the time of discharge. No drains were used during surgery. Face taping was applied and kept for 20 days. Patients were advised to eat cold and soft meals for 10 days and to use protective glasses and eye drops for few days. After taping removal, a tailored physical therapy was started with muscle re-education and relaxation exercises, scar tissue massage aiming to reduce adhesions, and to improve power of the rotated muscle.

\section{Case Series}

After institutional review board approval, we queried the facial palsy plastic surgery database at out hospital to identify all patients who underwent facial reanimation procedures between 2015 and 2018. Patients who had complete facial palsy for more than 3 years and not eligible for microsurgical restoration were considered for the EMTL procedure. We evaluated all patients who underwent operative EMTL 
treatment performed by the first author from September 2015 to May 2018. The patients were analyzed retrospectively, with more than 1 years of follow-up, and were evaluated in terms of functional-aesthetic results and postoperative complications. Fourteen patients were enrolled in the study. Several facial nerve scoring methods have been introduced over the years to document facial nerve recovery. We use as standard in facial plastic clinic the Sunnybrook Facial Grading System. ${ }^{9,10}$

We registered sex, age, original issue, evolution, and complications in all cases. Physical examination, photographs, video, satisfaction questionnaires, and spontaneous movement were carefully recorded and discussed at $1,3,6$, and 12 months.

Informed consent was obtained, including permissions for images publication, from all patients and the study was approved by the institutional review committee and was performed in accordance with the ethical standards of the Declaration of Helsinki.

Mean age at the time of treatment was 60 years (range 51-72 years). There were 10 men and 4 women. They were all inveterate palsies with minimum 4 years from the initial injury. Acoustic neuroma surgery was the predominant cause of the disability. Four patients suffered from parotid surgery and one was a posttraumatic case. Two patients had some form of restorative surgery without significant improvement. Three patients wore gold lid implant with incipient extrusion. Five patients suffered from recurrent corneal ulcerations with mild consequences. The preoperative Sunnybrook score ranged from 0 to 5 and the postoperative score ranged from 30 to 65 . Spontaneous smile achievement was obtained in 10 patients and only mild restoration in one patient ( - Figs. 6-7). Two patients developed a postoperative cheek seroma with no adherence to the physiatrical regimen and consequently poor outcome. There was a case of intraoral extrusion of polydioxanone suture used to anchor the temporal tendon to the modiolo. The support was partially lost in this patient. The scar and static correction were satisfactory in all patients ( $\boldsymbol{- \text { Fig. }}$ 8). Eye protection was improved in all cases with some form of active blinking in 6 cases.
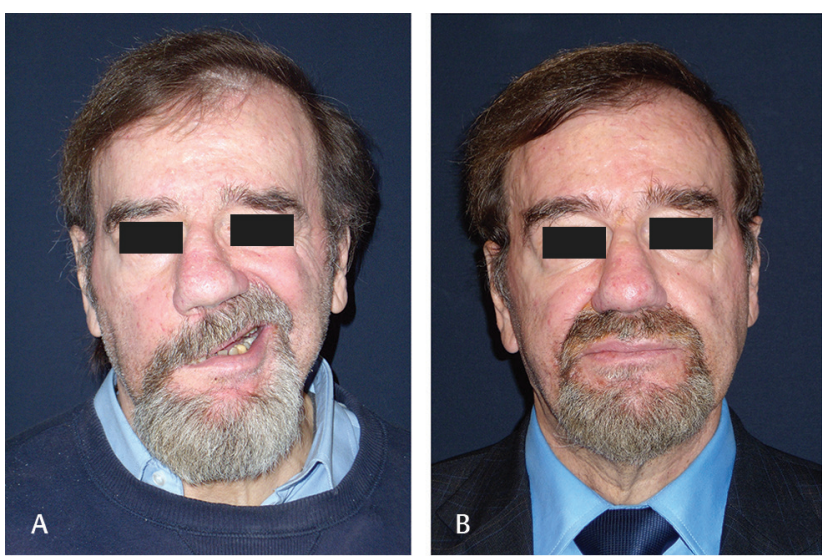

Fig. 6 Case series. (A, B) Evaluation preoperation (Sunnybrook composite score 8 ) and postoperation static at 6 months with very good symmetry.

\section{Discussion}

Gold standard treatment for inveterated facial palsy is functional muscle transfer with staged cross-facial nerve grafting. To date, results with the one-stage reconstruction with masseteric nerve as donor have been promising. We believe that one-stage method offers potential advantages in terms of safety, patient preferences, shorter total recovery time, and fewer resource implications. However, we support the concept described by Hontanilla et al, ${ }^{11}$ that is, "to recommend a free tissue transfer without offering the patient a chance of rehabilitation with simpler techniques may seem somewhat aggressive."
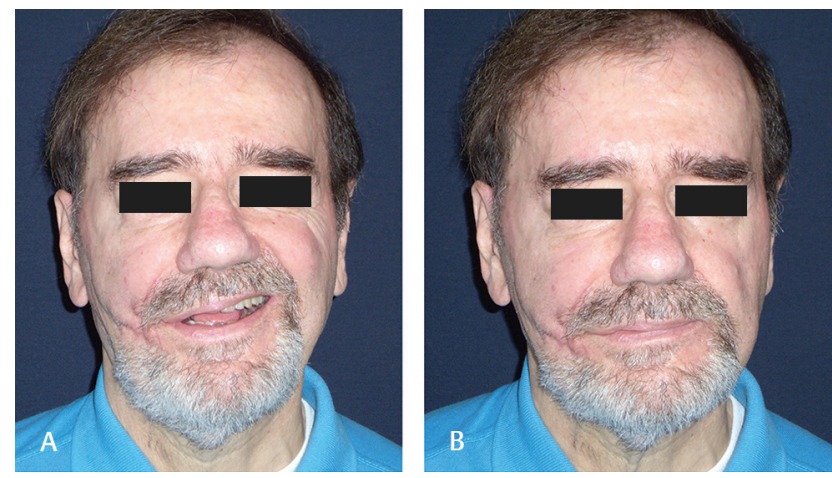

Fig. 7 Case series. (A, B) Dynamic evaluation at 3 months' follow-up with Sunnybrook composite score 75.
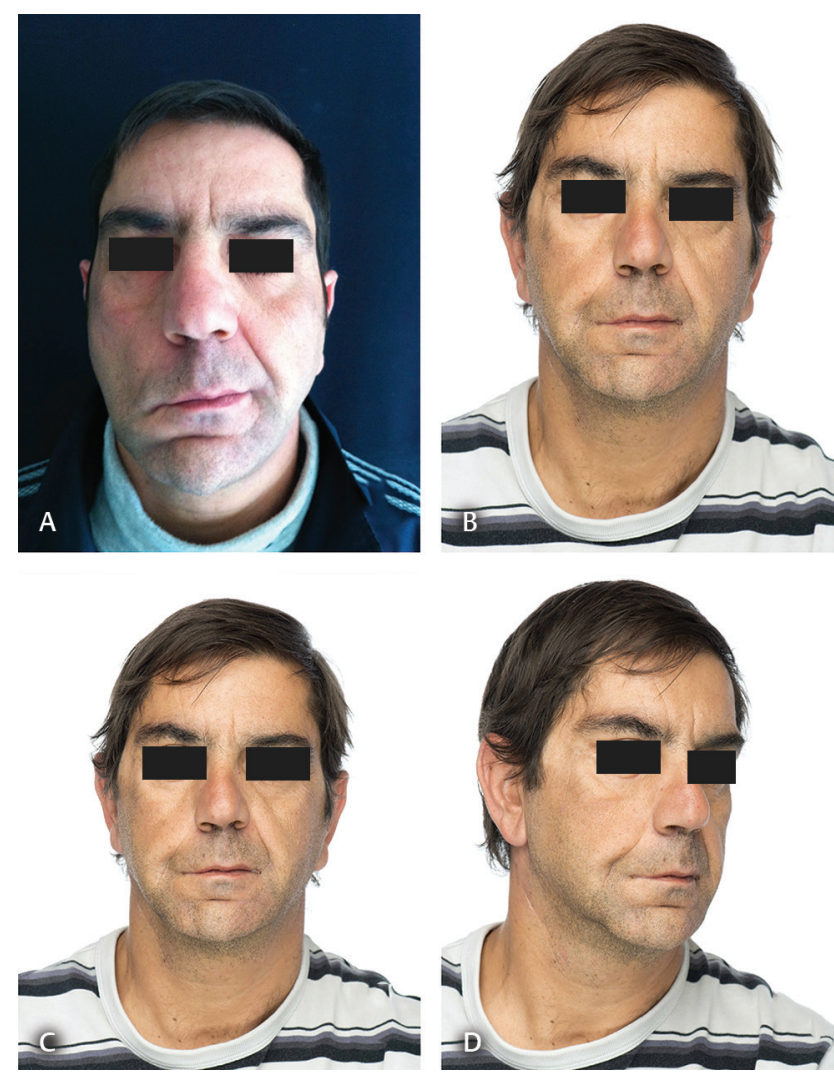

Fig. 8 Case series. (A, B) Static evaluation with closed eyes pre(composite Sunnybrook score 20) and postoperation (composite Sunnybrook score 60). (C, D) Postoperative scar appearance at 1-year follow-up in frontal and mid lateral view. 


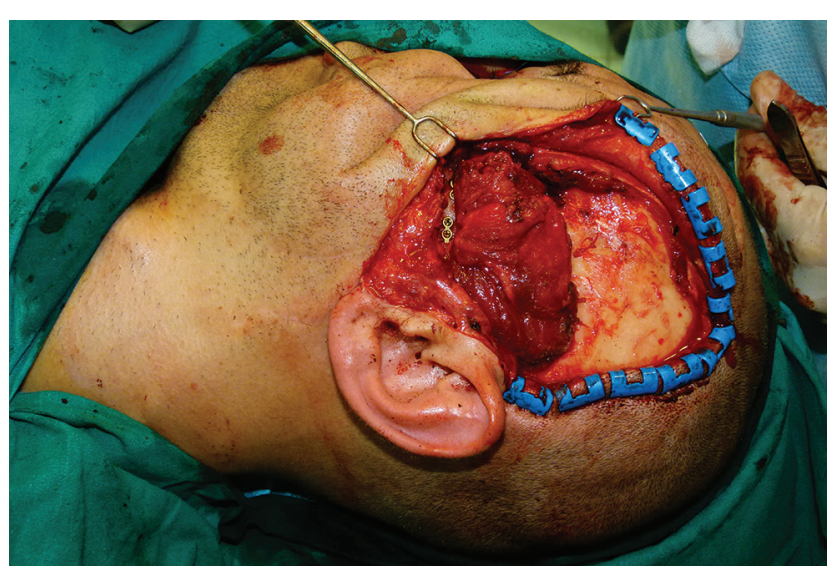

Fig. 9 Conventional Labbè approach.

We present a case series study with in-depth description of three critical surgical sites of intervention to approach all major components of the deformity and of the functional deficit of complete facial palsy. Several well-established different corrective techniques were conglobated in a single operation with minimal donor site morbidity and tiny residual scarring. Endoscopic assistance was imperative to minimize the "portals" for intervention and to reduce the complication rate. Conventional temporalis lengthening myoplasty described by Labbè et $\mathrm{al}^{12}$ has some drawbacks since it is quite invasive, as it requires a large temporal surgical site, osteotomy of the zygomatic arch, and a long incision in the nasolabial fold ( $\boldsymbol{-}$ Fig. 9). Nevertheless, new improvements of the Labbè technique have been already described showing the capacity to maintain benefits while reducing drawbacks. We started from this newer variation of the original technique pushing forward the reduction of the residual scar. Another consistent advantage in leaving temporalis muscle and its fascia undisturbed in temporal fossa is that it allows to use a narrow strip of the muscle separately for blink restoration.

Despite the central temporal muscle being used for reanimating the eyelids and the inferior tendon attachment to reanimate the lips, we have noticed only a mild synkinetic action, since the power of muscle contraction activates the lower tendon at a higher level. Nevertheless, it seems to be a drawback that is not perceived by the patient.

\section{Conclusion}

We present a reliable and reproducible surgical technique using a comprehensive endoscopic procedure with minimal donor-site morbidity to treat complete inveterate unilateral facial palsy. We have limited the description of this technique to inveterate facial palsy as alternative to microsurgical reconstruction. For other cases many options must be considered with particular attention to masseteric nerve transfer and cross-facial grafting.
This study showed that facial palsy correction with EMTL procedure offers a promising treatment alternative for patients with facial palsy not suitable for microsurgical muscle transposition. The EMTL procedure is a one-stage procedure with a wide therapeutic window since the rate of complications is low and the smile spontaneity can be restored in the majority of patients.

\section{Note}

Institutional review board approved the statement of conforming to the Declaration of Helsinki.

\section{Conflict of Interest}

No potential conflict of interest was reported by the authors.

\section{References}

1 Bos R, Reddy SG, Mommaerts MY. Lengthening temporalis myoplasty versus free muscle transfer with the gracilis flap for long-standing facial paralysis: a systematic review of outcomes. J Craniomaxillofac Surg 2016;44(8):940-951

2 Panossian A. Lengthening temporalis tyoplasty for single-stage smile reconstruction in children with facial paralysis. Plast Reconstr Surg 2016;137(4):1251-1261

3 Nduka C, Hallam MJ, Labbè D. Refinements in smile reanimation: 10-year experience with the lengthening temporalis myoplasty. J Plast Reconstr Aesthet Surg 2012;65(7):851-856

4 Moubayed SP, Labbé D, Rahal A. Lengthening temporalis myoplasty for facial paralysis reanimation: an objective analysis of each surgical step. JAMA Facial Plast Surg 2015;17(3):179-182

5 Nahai F, Saltz R, Endoscopic Plastic Surgery. 2nd ed. New York: Thieme Medical Publishers; 2008:chapter 4

6 Gupta AK, Jain S. Temporalis muscle sling revisited: a technique to restore ocular sphincter function. Ann Plast Surg 1994; 33(5):496-499

7 Boahene KD, Farrag TY, Ishii L, Byrne PJ. Minimally invasive temporalis tendon transposition. Arch Facial Plast Surg 2011; 13(1):8-13

8 Conley J, Baker DC, Selfe RW. Paralysis of the mandibular branch of the facial nerve. Plast Reconstr Surg 1982;70(5):569-577

9 Fattah AY, Gurusinghe ADR, Gavilan J, et al; Sir Charles Bell Society. Facial nerve grading instruments: systematic review of the literature and suggestion for uniformity. Plast Reconstr Surg 2015;135(2):569-579

10 Berner JE, Kamalathevan P, Kyriazidis I, Nduka C. Facial synkinesis outcome measures: A systematic review of the available grading systems and a Delphi study to identify the steps towards a consensus. J Plast Reconstr Aesthet Surg 2019; 72(6):946-963

11 Hontanilla B, Olivas J, Cabello Á, Marré D. Cross-face nerve grafting versus masseteric-to-facial nerve transposition for reanimation of incomplete facial paralysis: a comparative study using the FACIAL CLIMA evaluating system. Plast Reconstr Surg 2018;142(2):179e-191e

12 Labbè D, Bussu F, Iodice A. A comprehensive approach to long-standing facial paralysis based on lengthening temporalis myoplasty. Acta Otorhinolaryngol Ital 2012;32(3):145-153 